UDC: 615.065:618.3]:340.13 615.065:618.63]:340.13

DOI: 10.33320/maced.pharm.bull.2020.66.03.033

Short communication

\title{
Legal framework for pharmacovigilance in pregnancy and breastfeeding
}

\author{
Marijana Danevska ${ }^{1}$, Iskra Pechijareva Sadikarijo ${ }^{1}$, Julijana Sekovska ${ }^{2}$, \\ Katerina Ancevska Netkovska ${ }^{3}$, Zorica Naumovska ${ }^{3}$ \\ ${ }^{1}$ Agency for drugs and medical devices, Blvd. Ss. Cyril and Methodius 54, 1000 Skopje, R.N. Macedonia \\ ${ }^{2}$ Carso International, St. 34 No 5, 1000 Skopje, R.N. Macedonia \\ ${ }^{3}$ Faculty of Pharmacy, Ss. Cyril and Methodius University, Mother Theresa 47, 1000 Skopje, R.N. Macedonia
}

\section{Introduction}

Most women undergo medical treatment during pregnancy, irrespectively if the medicine is recommended for a given period or the treatment is unavoidable due to a particular illness. Scientifically based data on the safe use of medicines during pregnancy are an important public health need. In line with this, arise the inevitable need for establishment of pharmacovigilance guidance for the use of medicines during pregnancy on global and national level. The use of medicines during breastfeeding is also an area where further guidance and recommendations are needed. Additionally, important attention should be paid to OTC medicines, especially herbal drugs that are widely used even in the period of pregnancy and breastfeeding and are usually treated as "natural and safe "medicines by the patients.

. Spontaneous reporting of adverse reactions is insufficient to routinely detect fetal risks caused by medicine use during pregnancy. Because of limited data on the teratogenic potential of drugs prior to their marketing, post-marketing surveillance of medicine use in pregnancy is of particular importance in detecting medicines-induced fetal risks.

\section{Materials and methods}

Relevant European, American and Macedonian legislation was reviewed, in particular, Directive 2010/84/EU, Regulative (EU) 1235/2010, rulebooks, as well as PubMed, Medline and other relevant web sites for articles with empirical analysis, evaluating the impact of European and non-European regulatory activities.

\section{Results and discussion}

Since exposure to contraindicated medicines during pregnancy is sometimes unavoidable in each trimester, a safety monitoring mechanism needs to be established to provide reliable information to promote safe and effective treatment during pregnancy. Research involving the treatment of pregnant and breast-feeding women raises ethical and scientific issues. The safety data obtained in the pre-marketing phase of drug development are limited due to the limitations of the clinical trials regarding the size, timing and duration of subsequent monitoring as well as strictly defined inclusion and exclusion criteria of conducted studies (GVP, 2019). Pregnant and breast-feeding women are considered as a special population and are usually excluded from medicine development programs and clinical

\footnotetext{
*marijana.danevska@malmed.gov.mk
} 
trials, leading to gaps in knowledge when medicines are marketed. It is not known whether effective exposure is achieved during standard-dose pregnancy as in a non-pregnant adult woman and to what extent the medicine crosses the placenta and is excreted in breast milk. Pregnancy is associated with a wide range of physiological, anatomical and biochemical changes that significantly influence the pharmacokinetics of medicines (Pariente et al., 2016). Gathering data to gain a better understanding of the risks associated with such use and identifying and characterizing the risks is important even when no safety concerns have arisen before the premarketing phase. Timely and appropriate data collection and assessment enables patients and healthcare professionals to receive relevant information in a timely manner in order to make appropriate decisions about medicine use during pregnancy. The guiding principle is to minimize adverse reactions associated with medicine use during pregnancy.

Successful recruitment of an adequate number of pregnancies exposed to medicines, their comprehensive monitoring and complete and accurate determination of pregnancy outcomes are key elements in creating a well-designed registry or database. Accordingly, the aim is to build capacity for improved maternal and neonatal care within the overall health care system (Gliklich et al., 2018)

This mechanism will help overcome the deficiency commonly seen in medical practice when treating a pregnant woman with ineffective medicines due to potential safety concerns. It can also help assess medicines that are not recommended during pregnancy and which are sometimes unavoidable to save the mother or unborn child. Hence the need to create an appropriate pharmacovigilance guide during pregnancy.

\section{Conclusion}

Limited information for drug utilization during pregnancy and breastfeeding rice the need of implementation of additional legal framework in order to obtain safe drug treatment in this special population with positive benefit/risk ratio for the mother and unborn child.
These legal documents in the national level should be harmonized with regulation and rulebooks for good pharmacovigilance practice in pregnancy and breastfeeding already approved and implemented in by EMA.

\section{References}

Directive 2010/84/EU of the European Parliament and of the Council of 15 December 2010.

Gliklich, R.E., Dreyer, N.A., Leavy, M.B., Christian, J.B., 2018. Registries for evaluating patient outcomes: A user's guide, third ed. Addendum. Available at: https://effectivehealthcare.ahrq.gov/sites/default/files/ pdf/registries-guide-3rd-ed-addendum-research2018-revised_0.pdf (Last accessed: 25.3.2020).

Good Pharmacovigilance Practices and Pharmacoepidemiologic Assessment. Available at: https://www.fda.gov/media/71546/download (Last accessed: 25.3.2020).

Guidelines on good pharmacovigilance practices (GVP), 2019. Introductory cover note, last updated with chapter P. III on pharmacovigilance for the use of medicines by pregnant and breastfeeding women. Last updated: 11.122019.

Guideline on the exposure to medical products during pregnancy: need for post-authorization data. London, 14 November 2005, EMEA/CHMP/313666/2005.

Guidance for industry: Establishing Pregnancy Exposure Registries. Available at:

http://www.pregmedic.org/files/FDA GuidancePregancy Registries_Aug_2002.pdf (Last accessed: 25.3.2020).

Medicinal products and medical devices Act Official Gazette No. 106/07. Available at: https://malmed.gov.mk.

Regulation (EU) 1235/2010 of the European Parliament and of the Council of 15 December 2010.

Pariente, G., Leibson, T., Carls, A., Adams-Webber, T., Ito, S., Koren, G., 2016. Pregnancy- associated changes in pharmacokinetics: a systematic review. PLoS Med. 13(11), e1002160. Available at: https://www.ncbi.nlm.nih.gov/pubmed/2780221 (Last accessed: 25.3.2020).

Pharmacokinetics in pregnancy - study design, data analysis, and impact on dosing and labeling. Available at: https://www.fda.gov/media/71353 (Last accessed: 25.3.2020). 